\title{
Kernels of Directed Graph Laplacians
}

\author{
J. S. Caughman and J. J. P. Veerman \\ Department of Mathematics and Statistics \\ Portland State University \\ PO Box 751, Portland, OR 97207. \\ caughman@pdx.edu, veerman@pdx.edu
}

Submitted: Oct 28, 2005; Accepted: Mar 14, 2006; Published: Apr 11, 2006

Mathematics Subject Classification: 05C50

\begin{abstract}
Let $G$ denote a directed graph with adjacency matrix $Q$ and indegree matrix $D$. We consider the Kirchhoff matrix $L=D-Q$, sometimes referred to as the directed Laplacian. A classical result of Kirchhoff asserts that when $G$ is undirected, the multiplicity of the eigenvalue 0 equals the number of connected components of $G$. This fact has a meaningful generalization to directed graphs, as was observed by Chebotarev and Agaev in 2005. Since this result has many important applications in the sciences, we offer an independent and self-contained proof of their theorem, showing in this paper that the algebraic and geometric multiplicities of 0 are equal, and that a graphtheoretic property determines the dimension of this eigenspace - namely, the number of reaches of the directed graph. We also extend their results by deriving a natural basis for the corresponding eigenspace. The results are proved in the general context of stochastic matrices, and apply equally well to directed graphs with non-negative edge weights.
\end{abstract}

Keywords: Kirchhoff matrix. Eigenvalues of Laplacians. Graphs. Stochastic matrix.

\section{Definitions}

Let $G$ denote a directed graph with vertex set $V=\{1,2, \ldots, N\}$ and edge set $E \subseteq$ $V \times V$. To each edge $u v \in E$, we allow a positive weight $\omega_{u v}$ to be assigned. The adjacency matrix $Q$ is the $N \times N$ matrix whose rows and columns are indexed by the vertices, and where the $i j$-entry is $\omega_{j i}$ if $j i \in E$ and zero otherwise. The in-degree matrix $D$ is the $N \times N$ diagonal matrix whose $i i$-entry is the sum of the entries of the $i^{\text {th }}$ row of $Q$. The matrix $L=D-Q$ is sometimes referred to as the Kirchhoff matrix, and sometimes as the directed graph Laplacian of $G$.

A variation on this matrix can be defined as follows. Let $D^{+}$denote the pseudo-inverse of $D$. In other words, let $D^{+}$be the diagonal matrix whose $i i$-entry is $D_{i i}^{-1}$ if $D_{i i} \neq 0$ 
and whose $i i$-entry is zero if $D_{i i}=0$. Then the matrix $\mathcal{L}=D^{+}(D-Q)$ has nonnegative diagonal entries, nonpositive off-diagonal entries, all entries between -1 and 1 (inclusive) and all row sums equal to zero. Furthermore, the matrix $S=I-\mathcal{L}$ is stochastic.

We shall see (in Section 4) that both $L$ and $\mathcal{L}$ can be written in the form $D-D S$ where $D$ is an appropriately chosen nonnegative diagonal matrix and $S$ is stochastic. We therefore turn our attention to the properties of these matrices for the statement of our main results.

We show that for any such matrix $M=D-D S$, the geometric and algebraic multiplicities of the eigenvalue zero are equal, and we find a basis for this eigenspace (the kernel of $M$ ). Furthermore, the dimension of this kernel and the form of these eigenvectors can be described in graph theoretic terms as follows.

We associate with the matrix $M$ a directed graph $G$, and write $j \rightsquigarrow i$ if there exists a directed path from vertex $j$ to vertex $i$. For any vertex $j$, we define the reachable set $\mathcal{R}(j)$ to be the set containing $j$ and all vertices $i$ such that $j \rightsquigarrow i$. A maximal reachable set will be called a reach. We prove that the algebraic and geometric multiplicity of 0 as an eigenvalue for $M$ equals the number of reaches of $G$.

We also describe a basis for the kernel of $M$ as follows. Let $\mathcal{R}_{1}, \ldots \mathcal{R}_{k}$ denote the reaches of $G$. For each reach $\mathcal{R}_{i}$, we define the exclusive part of $\mathcal{R}_{i}$ to be the set $H_{i}=\mathcal{R}_{i} \backslash \cup_{j \neq i} \mathcal{R}_{j}$. Likewise, we define the common part of $\mathcal{R}_{i}$ to be the set $C_{i}=\mathcal{R}_{i} \backslash H_{i}$. Then for each reach $\mathcal{R}_{i}$ there exists a vector $v_{i}$ in the kernel of $M$ whose entries satisfy: (i) $\left(v_{i}\right)_{j}=1$ for all $j \in H_{i}$; (ii) $0<\left(v_{i}\right)_{j}<1$ for all $j \in C_{i}$; (iii) $\left(v_{i}\right)_{j}=0$ for all $j \notin \mathcal{R}_{i}$. Taken together, these vectors $v_{1}, v_{2}, \ldots, v_{k}$ form a basis for the kernel of $M$ and sum to the all 1's vector 1 .

Due to the recent appearance of Agaev and Chebotarev's notable paper [1], we would like to clarify the connections to their results. In that paper, the matrices studied have the form $M=\alpha(I-S)$ where $\alpha$ is positive and $S$ stochastic. A simple check verifies that this is precisely the set of matrices of the form $D-D S$, where $D$ is nonnegative diagonal. The number of reaches corresponds, in that paper, with the in-forest dimension. And where that paper concentrates on the location of the Laplacian eigenvalues in the complex plane, we instead have derived the form of the associated eigenvectors.

\section{Stochastic matrices}

A matrix is said to be (row) stochastic if the entries are nonnegative and the row sums all equal 1. Our first result is a special case of Geršgorin's theorem [3, p.344].

2.1 Lemma. Suppose $S$ is stochastic. Then each eigenvalue $\lambda$ satisfies $|\lambda| \leq 1$.

2.2 Definition. Given any real $N \times N$ matrix $M$, we denote by $G_{M}$ the directed graph with vertices $1, \ldots, N$ and an edge $j \rightarrow i$ whenever $M_{i j} \neq 0$. For each vertex $i$, set

$\mathcal{N}_{i}:=\{j \mid j \rightarrow i\}$. We write $j \rightsquigarrow i$ if there exists a directed path in $G_{M}$ from vertex $j$ to vertex $i$. Furthermore, for any vertex $j$, we define $\mathcal{R}(j)$ to be the set containing $j$ and all vertices $i$ such that $j \rightsquigarrow i$. We refer to $\mathcal{R}(j)$ as the reachable set of vertex $j$. Finally, we say a matrix $M$ is rooted if there exists a vertex $r$ in $G_{M}$ such that $\mathcal{R}(r)$ contains every vertex of $G_{M}$. We refer to such a vertex $r$ as a root. 
2.3 Lemma. Suppose $S$ is stochastic and rooted. Then the eigenspace $\mathcal{E}_{1}$ associated with the eigenvalue 1 is spanned by the all-ones vector 1.

Proof. Conjugating $S$ by an appropriate permutation matrix if necessary, we may assume that vertex 1 is a root. Since $S$ is stochastic, $S \mathbf{1}=\mathbf{1}$ so $\mathbf{1} \in \mathcal{E}_{1}$. By way of contradiction, suppose $\operatorname{dim}\left(\mathcal{E}_{1}\right)>1$ and choose linearly independent vectors $x, y \in \mathcal{E}_{1}$. Suppose $\left|x_{i}\right|$ is maximized at $i=n$. Comparing the $n$-entry on each side of the equation $x=S x$, we see that

$$
\left|x_{n}\right| \leq \sum_{j \in \mathcal{N}_{n}} S_{n j}\left|x_{j}\right| \leq\left|x_{n}\right| \sum_{j \in \mathcal{N}_{n}} S_{n j}=\left|x_{n}\right| .
$$

Therefore, equality holds throughout, and $\left|x_{j}\right|=\left|x_{n}\right|$ for all $j \in \mathcal{N}_{n}$. In fact, since $\sum_{j \in \mathcal{N}_{n}} S_{n j} x_{j}=x_{n}$, it follows that $x_{j}=x_{n}$ for all $j \in \mathcal{N}_{n}$. Since $S$ is rooted at vertex 1 , a simple induction now shows that $x_{1}=x_{n}$. So $\left|x_{i}\right|$ is maximized at $i=1$. The same argument applies to any vector in $\mathcal{E}_{1}$ and so $\left|y_{i}\right|$ is maximized at $i=1$.

Since $y_{1} \neq 0$ we can define a vector $z$ such that $z_{i}:=x_{i}-\frac{x_{1}}{y_{1}} y_{i}$ for each $i$. This vector $z$, as a linear combination of $x$ and $y$, must belong to $\mathcal{E}_{1}$. It follows that $\left|z_{i}\right|$ is also maximized at $i=1$. But $z_{1}=0$ by definition, so $z_{i}=0$ for all $i$. It follows that $x$ and $y$ are not linearly independent, a contradiction.

2.4 Lemma. Suppose $S$ is stochastic $N \times N$ and vertex 1 is a root. Further assume $\mathcal{N}_{1}$ is empty. Let $P$ denote the principal submatrix obtained by deleting the first row and column of $S$. Then the spectral radius of $P$ is strictly less than 1.

Proof. Since $\mathcal{N}_{1}$ is empty, $S$ is block lower-triangular with $P$ as a diagonal block. So the spectral radius of $P$ cannot exceed that of $S$. Therefore, by Lemma 2.1, the spectral radius of $P$ is at most 1. By way of contradiction, suppose the spectral radius of $P$ is equal to 1 . Then by the Perron-Frobenius theorem (see [3, p. 508]), we would have $P x=x$ for some nonzero vector $x$.

Define a vector $v$ with $v_{1}=0$ and $v_{i}=x_{i-1}$ for $i \in\{2, \ldots, N\}$. We find that

$$
S v=\left(\begin{array}{c|c}
1 & 0 \cdots 0 \\
\hline S_{21} & \\
\vdots & P \\
S_{N 1} &
\end{array}\right)\left(\begin{array}{c}
0 \\
x
\end{array}\right)=\left(\begin{array}{c}
0 \\
x
\end{array}\right)=v
$$

So $v \in \mathcal{E}_{1}$. But $v_{1}=0$, so Lemma 2.3 implies $x=0$. This contradiction completes the proof.

2.5 Corollary. Suppose $S$ is stochastic and $N \times N$. Assume the vertices of $G_{S}$ can be partitioned into nonempty sets $A, B$ such that for every $b \in B$, there exists $a \in A$ with $a \rightsquigarrow b$ in $G_{S}$. Then the spectral radius of the principal submatrix $S_{B B}$ obtained by deleting from $S$ the rows and columns of $A$ is strictly less than 1 .

Proof. Define the matrix $\hat{S}$ by

$$
\hat{S}=\left(\begin{array}{cc}
1 & 0 \\
\mathbf{u} & S_{B B}
\end{array}\right)
$$


where $\mathbf{u}$ is chosen so that $\hat{S}$ is stochastic. We claim that $\hat{S}$ is rooted (at 1 ). To see this, pick any $b \in B$. We must show $1 \rightsquigarrow b$ in $G_{\hat{S}}$. By hypothesis there exists $a \in A$ with $a \rightsquigarrow b$ in $G_{S}$. Let

$$
a=x_{0} \rightarrow x_{1} \rightarrow \cdots \rightarrow x_{n}=b
$$

be a directed path in $G_{S}$ from $a$ to $b$. Let $i$ be maximal such that $x_{i} \in A$. Then the $x_{i+1}, x_{i}$ entry of $S$ is nonzero, so the $x_{i+1}$ row of $S_{B B}$ has row sum strictly less than 1 . Therefore, the $x_{i+1}$ entry of the first column of $\hat{S}$ is nonzero. So $1 \rightarrow x_{i+1}$ in $G_{\hat{S}}$ and therefore $1 \rightsquigarrow b$ in $G_{\hat{S}}$ as desired. So $\hat{S}$ is rooted, and the previous lemma gives the result.

2.6 Definition. A set $\mathcal{R}$ of vertices in a graph will be called a reach if it is a maximal reachable set; in other words, $\mathcal{R}$ is a reach if $\mathcal{R}=\mathcal{R}(i)$ for some $i$ and there is no $j$ such that $\mathcal{R}(i) \subset \mathcal{R}(j)$ (properly). Since our graphs all have finite vertex sets, such maximal sets exist and are uniquely determined by the graph. For each reach $\mathcal{R}_{i}$ of a graph, we define the exclusive part of $\mathcal{R}_{i}$ to be the set $H_{i}=\mathcal{R}_{i} \backslash \cup_{j \neq i} \mathcal{R}_{j}$. Likewise, we define the common part of $\mathcal{R}_{i}$ to be the set $C_{i}=\mathcal{R}_{i} \backslash H_{i}$.

2.7 Theorem. Suppose $S$ is stochastic $N \times N$ and let $\mathcal{R}$ denote a reach of $G_{S}$ with exclusive part $H$ and common part $C$. Then there exists an eigenvector $v \in \mathcal{E}_{1}$ whose entries satisfy

(i) $v_{i}=1 \quad$ for all $i \in H$,

(ii) $0<v_{i}<1 \quad$ for all $i \in C$,

(iii) $0 \quad$ for all $i \notin \mathcal{R}$.

Proof. Let $Y$ denote the set of vertices not in $\mathcal{R}$. Permuting rows and columns of $S$ if necessary, we may write $S$ as

$$
S=\left(\begin{array}{ccc}
S_{H H} & S_{H C} & S_{H Y} \\
S_{C H} & S_{C C} & S_{C Y} \\
S_{Y H} & S_{Y C} & S_{Y Y}
\end{array}\right)=\left(\begin{array}{ccc}
S_{H H} & \mathbf{0} & \mathbf{0} \\
S_{C H} & S_{C C} & S_{C Y} \\
\mathbf{0} & \mathbf{0} & S_{Y Y}
\end{array}\right)
$$

Since $S_{H H}$ is a rooted stochastic matrix, it has eigenvalue 1 with geometric multiplicity 1. The associated eigenvector is $\mathbf{1}_{H}$.

Observe that $S_{C C}$ has spectral radius $<1$ by Corollary 2.5. Further, notice that $S\left(\mathbf{1}_{H}, \mathbf{0}_{C}, \mathbf{0}_{Y}\right)^{T}=\left(\mathbf{1}_{H}, S_{C H} \mathbf{1}_{H}, \mathbf{0}_{Y}\right){ }^{T}$ Using this, we find that solving the equation

$$
S\left(\mathbf{1}_{H}, \mathbf{x}, \mathbf{0}_{C}\right)^{T}=\left(\mathbf{1}_{H}, \mathbf{x}, \mathbf{0}_{C}\right)^{T}
$$

for $\mathbf{x}$ amounts to solving

$$
\left(\begin{array}{c}
\mathbf{1}_{H} \\
S_{C H} \mathbf{1}_{H}+S_{C C} \mathbf{x} \\
\mathbf{0}_{Y}
\end{array}\right)=\left(\begin{array}{c}
\mathbf{1}_{H} \\
\mathbf{x} \\
\mathbf{0}_{Y}
\end{array}\right)
$$


Solving the above, however, is equivalent to solving $\left(I-S_{C C}\right) \mathbf{x}=S_{C H} \mathbf{1}_{H}$. Since the spectral radius of $S_{C C}$ is strictly less than 1, the eigenvalues of $I-S_{C C}$ cannot be 0 . So $I-S_{C C}$ is invertible. It follows that $\mathbf{x}=\left(I-S_{C C}\right)^{-1} S_{C H} \mathbf{1}_{H}$ is the desired solution.

Conditions (i) and (iii) are clearly satisfied by $\left(\mathbf{1}_{H}, \mathbf{x}, \mathbf{0}_{Y}\right){ }^{T}$ so it remains only to verify (ii). To see that the entries of $\mathbf{x}$ are positive, note that $\left(I-S_{C C}\right)^{-1}=\sum_{i=0}^{\infty} S_{C C}^{i}$, so the entries of $\mathbf{x}$ are nonnegative and strictly less than 1 . But every vertex in $C$ has a path from the root, where the eigenvector has value 1 . So since each entry in the eigenvector for $S$ must equal the average of the entries corresponding to its neighbors in $G_{S}$, all entries in $C$ must be positive.

\section{Matrices of the form $D-D S$}

We now consider matrices of the form $D-D S$ where $D$ is a nonnegative diagonal matrix and $S$ is stochastic. We will determine the algebraic multiplicity of the zero eigenvalue. We begin with the rooted case.

3.1 Lemma. Suppose $M=D-D S$, where $D$ is a nonnegative diagonal matrix and $S$ is stochastic. Suppose $M$ is rooted. Then the eigenvalue 0 has algebraic multiplicity 1.

Proof. Let $M=D-D S$ be given as stated. First we claim that, without loss of generality, $S_{i i}=1$ whenever $D_{i i}=0$. To see this, suppose $D_{i i}=0$ for some $i$. If $S_{i i} \neq 1$, let $S^{\prime}$ be the stochastic matrix obtained by replacing the $i^{\text {th }}$ row of $S$ by the $i^{\text {th }}$ row of the identity matrix $I$, and let $M^{\prime}=D-D S^{\prime}$. Observe that $M=M^{\prime}$, and this proves our claim. So we henceforth assume that

$$
S_{i i}=1 \quad \text { whenever } \quad D_{i i}=0 \text {. }
$$

Next we claim that, given $(1), \operatorname{ker}(M)$ must be identical with $\operatorname{ker}(I-S)$. To see this, note that if $(I-S) v=0$ then clearly $M v=D(I-S) v=0$. Conversely, suppose $M v=0$. Then $D(I-S) v=0$ so the vector $w=(I-S) v$ is in the kernel of $D$. If $w$ has a nonzero entry $w_{i}$ then $D_{i i}=0$. Recall this implies $S_{i i}=1$ and the $i^{\text {th }}$ row of $I-S$ is zero. But $w=(I-S) v$, so $w_{i}$ must be zero. This contradiction implies $w$ must have no nonzero entries, and therefore $(I-S) v=0$. So $M$ and $I-S$ have identical nullspaces as desired.

By Lemma 2.3, $S \mathbf{1}=\mathbf{1}$, so $M \mathbf{1}=0$. Therefore the geometric multiplicity, and hence the algebraic multiplicity, of the eigenvalue 0 must be at least 1 . By way of contradiction, suppose the algebraic multiplicity is greater than 1 . Then there must be a nonzero vector $x$ and an integer $d \geq 2$ such that

$$
M^{d-1} x \neq 0 \quad \text { and } \quad M^{d} x=0 .
$$

Now, since $\operatorname{ker} M=\operatorname{ker}(I-S)$, Lemma 2.3 and the above equation imply that $M^{d-1} x$ must be a multiple of the vector 1 . Scaling $M^{d-1} x$ appropriately, we find there exists a vector $v$ such that

$$
M v=-\mathbf{1}
$$


Suppose $\operatorname{Re}\left(v_{i}\right)$ is maximized at $i=n$. Comparing the $n$-entries above, we find

$$
D_{n n} \operatorname{Re}\left(v_{n}\right)+1=D_{n n} \sum_{j \in \mathcal{N}_{n}} S_{n j} \operatorname{Re}\left(v_{j}\right) \leq D_{n n} \operatorname{Re}\left(v_{n}\right) \sum_{j \in \mathcal{N}_{n}} S_{n j}=D_{n n} \operatorname{Re}\left(v_{n}\right),
$$

which is clearly impossible.

3.2 Theorem. Suppose $M=D-D S$, where $D$ is a nonnegative diagonal matrix and $S$ is stochastic. Then the number of reaches of $G_{M}$ equals the algebraic and geometric multiplicity of 0 as an eigenvalue of $M$.

Proof. Let $\mathcal{R}_{1}, \ldots, \mathcal{R}_{k}$ denote the reaches of $G_{M}$ and let $H_{i}$ denote the exclusive part of $\mathcal{R}_{i}$ for each $1 \leq i \leq k$, and let $C=\cup_{i=1}^{k} C_{i}$ denote the union of the common parts of all the reaches. Simultaneously permuting the rows and columns of $M, D$, and $S$ if necessary, we may write $M=D-D S$ as

$$
M=\left(\begin{array}{cccc|c}
D_{H_{1} H_{1}}\left(I-S_{H_{1} H_{1}}\right) & \mathbf{0} & \cdots & \mathbf{0} & \mathbf{0} \\
\mathbf{0} & \ddots & \cdots & \mathbf{0} & \mathbf{0} \\
\vdots & \vdots & \ddots & \vdots & \vdots \\
\mathbf{0} & \mathbf{0} & \cdots & D_{H_{k} H_{k}}\left(I-S_{H_{k} H_{k}}\right) & \mathbf{0} \\
\hline-D_{C C} S_{C H_{1}} & \cdots & \cdots & -D_{C C} S_{C H_{k}} & D_{C C}\left(I-S_{C C}\right)
\end{array}\right)
$$

The characteristic polynomial $\operatorname{det}(M-\lambda I)$ is therefore given by

$$
\operatorname{det}\left(D_{H_{1} H_{1}}\left(I-S_{H_{1} H_{1}}\right)-\lambda I\right) \cdots \operatorname{det}\left(D_{H_{k} H_{k}}\left(I-S_{H_{k} H_{k}}\right)-\lambda I\right) \cdot \operatorname{det}\left(D_{C C}\left(I-S_{C C}\right)-\lambda I\right) .
$$

By Lemma 3.1, each submatrix $D_{H_{1} H_{1}}\left(I-S_{H_{1} H_{1}}\right)$ has eigenvalue 0 with algebraic and geometric multiplicity 1 . But observe that $D_{C C}$ has nonzero diagonal entries since $C$ is the union of the common parts $C_{i}$, so $D_{C C}\left(I-S_{C C}\right)$ is invertible by Corollary 2.5. The theorem now follows.

We now offer the following characterization of the nullspace.

3.3 Theorem. Suppose $M=D-D S$, where $D$ is a nonnegative $N \times N$ diagonal matrix and $S$ is stochastic. Suppose $G_{M}$ has $k$ reaches, denoted $\mathcal{R}_{1}, \ldots, \mathcal{R}_{k}$, where we denote the exclusive and common parts of each $\mathcal{R}_{i}$ by $H_{i}, C_{i}$ respectively. Then the nullspace of $M$ has a basis $\gamma_{1}, \gamma_{2}, \ldots, \gamma_{k}$ in $\mathbb{R}^{N}$ whose elements satisfy:

(i) $\gamma_{i}(v)=0$ for $v \notin \mathcal{R}_{i}$;

(ii) $\gamma_{i}(v)=1$ for $v \in H_{i}$;

(iii) $\gamma_{i}(v) \in(0,1)$ for $v \in C_{i}$;

(iv) $\sum_{i} \gamma_{i}=\mathbf{1}_{N}$ 
Proof. Let $M=D-D S$ be given as stated. As in the proof of Theorem 3.2 above, we may assume without loss of generality that

$$
S_{i i}=1 \quad \text { whenever } \quad D_{i i}=0 .
$$

We further observe, as in the proof of Theorem 3.2, that $M$ and $I-S$ have identical nullspaces, given (2).

Notice that the diagonal entries of a matrix do not affect the reachable sets in the associated graph, so the reaches of $G_{I-S}$ are identical with the reaches of $G_{S}$. Furthermore, scaling rows by nonzero constants also leaves the corresponding graph unchanged, so $G_{M}=G_{D(I-S)}=G_{I-S}$. Therefore the reaches of $G_{M}$ are identical with the reaches of $G_{S}$.

Applying Theorems 2.7 and 3.2, we find that the nullity of the matrix $M$ equals $k$ and the nullspace of $M$ has a basis satisfying (i)-(iii). To see (iv), observe that the all 1's vector $\mathbf{1}$ is a null vector for $M$, and notice that the only linear combination of these basis vectors that assumes the value 1 on each of the $H_{i}$ is their sum.

\section{Graph Laplacians}

In this section, we simply apply our results to the Laplacians $L$ and $\mathcal{L}$ of a (weighted, directed) graph, as discussed in Section 1.

4.1 Corollary. Let $G$ denote a weighted, directed graph and let $\mathcal{L}$ denote the (directed) Laplacian matrix $\mathcal{L}=D^{+}(D-Q)$. Suppose $G$ has $N$ vertices and $k$ reaches. Then the algebraic and geometric multiplicity of the eigenvalue 0 equals $k$. Furthermore, the associated eigenspace has a basis $\gamma_{1}, \gamma_{2}, \ldots, \gamma_{k}$ in $\mathbb{R}^{N}$ whose elements satisfy: (i) $\gamma_{i}(v)=0$ for $v \in G-\mathcal{R}_{i}$; (ii) $\gamma_{i}(v)=1$ for $v \in H_{i}$; (iii) $\gamma_{i}(v) \in(0,1)$ for $v \in C_{i}$; (iv) $\sum_{i} \gamma_{i}=\mathbf{1}_{N}$.

Proof. The matrix $S=I-\mathcal{L}$ is stochastic and the graphs $G$ and $G_{S}$ have identical reaches. The result follows by applying Theorem 3.3.

We next observe that the same results hold for the Kirchhoff matrix $L=D-Q$.

4.2 Corollary. Let $G$ denote a directed graph and let $L$ denote the Kirchhoff matrix $L=D-Q$. Suppose $G$ has $N$ vertices and $k$ reaches. Then the algebraic and geometric multiplicity of the eigenvalue 0 equals $k$. Furthermore, the associated eigenspace has a basis $\gamma_{1}, \gamma_{2}, \ldots, \gamma_{k}$ in $\mathbb{R}^{N}$ whose elements satisfy: (i) $\gamma_{i}(v)=0$ for $v \in G-\mathcal{R}_{i}$; (ii) $\gamma_{i}(v)=1$ for $v \in H_{i}$; (iii) $\gamma_{i}(v) \in(0,1)$ for $v \in C_{i}$; (iv) $\sum_{i} \gamma_{i}=\mathbf{1}_{N}$.

Proof. One simply checks that the matrix $L$ has the form $D-D S$ where $S$ is the stochastic matrix $I-\mathcal{L}$ from above, and $D$ is the in-degree matrix of $G$. The result follows by applying Theorem 3.3.

In numerous applications, in particular those related to difference - or differential equations (see [6]), it is a crucial fact that any nonzero eigenvalue of the Laplacian has a strictly positive real part. Using some of the stratagems already exhibited, the proof of this fact is easy, and we include the result for completeness. 
4.3 Theorem. Any nonzero eigenvalue of a Laplacian matrix of the form $D-D S$, where $D$ is nonnegative diagonal and $S$ is stochastic, has (strictly) positive real part.

Proof. Let $\lambda \neq 0$ be an eigenvalue of $D-D S$ and $v$ a corresponding eigenvector, so $(D-D S) v=\lambda v$. Thus for all $i$,

$$
D_{i i} v_{i}=\lambda v_{i}+D_{i i} \sum_{j} S_{i j} v_{j}
$$

Suppose $D_{i i}$ is zero. Then $\lambda v_{i}=0$. Since $\lambda \neq 0$ it follows that $v_{i}=0$. Since $\lambda \neq 0$, the vector $v$ is not a multiple of $\mathbf{1}$. Let $n$ be such that $\left|v_{i}\right|$ is maximized at $i=n$. Multiply $v$ by a nonzero complex number so that $v_{n}$ is real. Since $v_{n}$ is nonzero, the above argument shows that $D_{n n} \neq 0$. Dividing (3) for $i=n$ by $D_{n n}$ and taking the real and imaginary parts separately, we obtain

$$
\sum_{j} S_{n j} \operatorname{Re}\left(v_{j}\right)=\left(1-\frac{\operatorname{Re}(\lambda)}{D_{n n}}\right) v_{n}, \quad \sum_{j} S_{n j} \operatorname{Im}\left(v_{j}\right)=-\frac{\operatorname{Im}(\lambda)}{D_{n n}} v_{n} .
$$

The first of these equations implies that $\operatorname{Re}(\lambda) \geq 0$. Now if $\operatorname{Re}(\lambda)=0$ then for all $j \in \mathcal{N}_{n}$ we have $v_{j}=v_{n}$ and thus $\operatorname{Im}\left(v_{j}\right)=0$. Notice that in this case, the imaginary part of $\lambda$ must be nonzero. So in the second equation above, the left hand side is zero but the right hand side is not. The conclusion is now immediate.

Acknowledgment. The authors would like to thank Gerardo Lafferriere and Anca Williams for many helpful discussions and insightful comments on this topic.

\section{References}

[1] R. Agaev and P. Chebotarev. On the spectra of nonsymmetric laplacian matrices. Linear Algebra App., 399:157-168, 2005.

[2] P. Chebotarev and R. Agaev. Forest matrices around the laplacian matrix. Linear Algebra App., 356:253-274, 2002.

[3] R. A. Horn and Charles R. Johnson. Matrix Analysis. Cambridge University Press, Cambridge, 1985.

[4] T. Leighton and R. L. Rivest. The markov chain tree theorem. Computer Science Technical Report MIT/LCS/TM-249, Laboratory of Computer Scinece, MIT, Cambridge, Mass., 1983.

[5] U. G. Rothblum. Computation of the eigenprojection of a nonnegative matrix at its spectral radius. Mathematical Programming Study, 6:188-201, 1976.

[6] J. J. P. Veerman, G. Lafferriere, J. S. Caughman, and A. Williams. Flocks and formations. J. Stat. Phys., 121:901-936, 2005. 